\title{
S-Nitrosoglutathione (GSNO) Mediates Brain Response to Hypoxia
}

\author{
A review of: Lipton AJ, Johnson MA, Macdonald T, et al. $2001 S$-nitrosothiols signal the ventilatory response to \\ hypoxia. Nature 413:171-174
}

$\mathrm{N}^{\mathrm{n}}$ ITRIC OXIDE (NंO) gas is a reactive nitrogen species routinely formed in combustion engines. In biological systems, it is synthesized from L-arginine by three isoforms of nitric oxide synthase in neurons (NOS1), macrophages (NOS2), and endothelium (NOS3). In addition to inducible NOS2, neuronal NOS1 and endothelial NOS3 are also inducible and feedback regulated. The activation of endothelial NOS3 in the cardiovascular system by acetylcholine leads to the release of $\dot{\mathrm{NO}}$ or the endothelium-derived relaxing factor (EDRF) that subsequently enters vascular smooth muscle triggering vasorelaxation (the 1998 Nobel Prize project of R.F. Furchgott, L.J. Ignarro, and F. Murad) via a cyclic GMP (cGMP)-dependent mechanism (1). Owing to the free radical property of the highly reactive unpaired electron on the nitrogen atom, $\mathrm{NO}$ is chemically reactive and biologically multifunctional (2). Medicinal $\dot{N O}$ gas has been recently approved as a treatment of persistent pulmonary hypertension in the United States and the European Union. Some of the new NंO donor agents (ie NCX 1015)developed by the NicOx S.A. are undergoing clinical trials.

The major chemical and biological effects of $\dot{\mathrm{NO}}$ are very intriguing (2). 1) $\dot{\mathrm{NO}}$ binds to the heme moiety to activate guanylyl cyclase for signaling through the cGMP-PKG pathway to induce vasorelaxation and neuroprotection. 2) $\mathrm{NO}$ also binds to iron and regulates the redox cycling of iron complexes including hemoglobin, iron regulating protein, and ferrous citrate. 3) $\dot{\mathrm{NO}}$ is an atypical antioxidant rather than a pro-oxidant since it scavenges cytotoxic reactive oxygen species and peroxyl lipid radicals. 4) $\dot{\mathrm{NO}}$ is a redox modulator for cysteinecontaining peptides (ie L-glutathione $/ \boldsymbol{\gamma}$ $\mathrm{GSH})$ because it readily reacts with the sulfhydryl group (-SH) of cysteine to form $S$-nitrosocysteine and/or thiol radical (ĠS) of GSH to form $S$-nitrosoglutathione (GSNO), respectively. Pharmacologically, inhalation of $\mathrm{NO}$ gas increases airway muscle relaxation. Some of the

\section{Chunang C. Chiueh}

inhaled and/or induced $\dot{N O}$ in the lung may convert to GSNO and other $S$ nitrosothiols that prevent platelet aggregation, facilitate the control of uptake and delivery of oxygen, and regulate the $\mathrm{N}$-methyl-D-aspartate (NMDA) ion channel $(3,4)$. Furthermore, GSNO is at least 3 to 5 orders of magnitude more potent than GSH against oxidative stress caused by peroxynitrite $\left(\mathrm{ONOO}^{-}\right)$(5). GSNO activates a cGMP-mediated synthesis of thioredoxin (TRX) that protects against oxidative stressinduced apoptosis for promoting preconditioning-induced compensatory hormesis or neuroprotection (6).

In the central nervous system GSNO can be generated during ischemia in 1) endothelial and 2) astroglial cells where micromolar levels of $\dot{\mathrm{NO}}$ and millimolar concentrations of GSH are found (7). GSNO is more stable in the biological system than its precursor $\mathrm{NO}$; it can enter neighboring neurons and be converted by $\gamma$-glutamyl transpeptidase $(\gamma$-GT) to $S$ nitrosocysteinyl glycine (CGSNO). CGSNO is the putative signaling molecule for regulating the ventilatory responses to hypoxia at the brain stem level of the nuclear tractus solitarius (NTS) (8) and for controlling sensory transmission in the ventrobasal thalamus (9). Lipton et al. (8) employed mass spectrometry to show that GSNO obtained from deoxygenated, but not oxygenated, blood when infused into NTS mimics the ventilatory effects of hypoxia. The generation of CGSNO in the brain regions has been reported by the research team leaded by Salt et al. (9). Either gene knockout or inhibition of $\gamma$-GT by acivicin prevents the physiological effects of GSNO in the NTS. These in vivo findings indicate that CGSNO is a signaling molecule in the brain stem for triggering a cGMPindependent feedback regulation of ventilation in the lung following hypoxia. These elegant results of Lipton et al. (8) fit well with a recent proposal of Chiueh and Rauhala (7) that GSNO and related $S$-nitrosothiols may serve as signaling molecules between endothelial or astroglial cells and brain neurons. The present article adds to the observations in a recent commentary (10) and together emphasizes and explains the importance of these findings. The studies of Lipton et al and prior $S$-nitrosothiol studies will be cited by physiology and pharmacology textbooks as key observations that advance our understanding of the pivotal signaling role of GSNO and related CGSNO in brain function, especially the cell to neuron communication for controlling the respiratory cycle.

\section{REFERENCES}

1. Murad F 1999 Cellular signaling with nitric oxide and cyclic GMP. Braz J Med Biol Res 32:1317-1327

2. Chiueh CC, Hong J-S, Leong S-K (eds) 2002 Nitric oxide: Novel actions, deleterious effects, and clinical potential. Ann N Y Acad Sci 962: (in press)

3. Stamler JS, Toone EJ, Lipton SA, Sucher NJ 1997 (S)NO signals: translocation, regulation, and a consensus motif. Neuron 18:691-696

4. Choi YB, Lipton SA 2000 Redox modulation of the NMDA receptor. Cell Mol Life Sci 57:1535-1541

5. Rauhala P, Lin AM-Y, Chiueh CC 1998 Neuroprotection by $S$-nitrosoglutathione of brain dopamine neurons from oxidative stress. FASEB J 12:165-173

6. Andoh T, Chock PB, Chiueh CC 2001 The roles of thioredoxin in protection against oxidative stressinduced apoptosis in SH-SY5Y cells. J Biol Chem 277: (in press)

7. Chiueh CC, Rauhala P 1999 The redox pathway of $S$-nitrosoglutathione, glutathione and nitric oxide in cell to neuron communications. Free Radic Res 31:641-650

8. Lipton AJ, Johnson MA, Macdonald T, Lieberman MW, Gozal D, Gaston B $2001 S$-nitrosothiols signal the ventilatory response to hypoxia. Nature 413:171-174

9. Salt TE, Zhang H, Mayer B, Benz B, Binns KE, Do KQ 2000 Novel mode of nitric oxide neurotransmission mediated via $S$-nitroso-cysteinyl-glycine. Eur J Neurosci 12:3919-3925

10. Lipton SA 2001 News and views in physiology: Nitric oxide and respiration. Nature 413:118-119

Laboratory of Clinical Science, NIMH, NIH, Building 10, Room 3D-41

Bethesda, MD 20892-1264 U.S.A.

chiueh@helix.nih.gov 